\title{
The association of BMI with risk of recurrence and progression in patients with non-muscle-invasive bladder cancer
}

\author{
Alina Vrieling \\ Radboud Institute for Health Sciences, Radboud University Medical Center, Nijmegen, The Netherlands \\ Correspondence to: Dr. Alina Vrieling. Radboud University Medical Center, Department for Health Evidence (Mailbox 133), P.O. Box 9101, 6500 HB \\ Nijmegen, The Netherlands. Email: Alina.Vrieling@radboudumc.nl. \\ Provenance: This is an Invited Editorial commissioned by Section Editor Xiao Li (Department of Urology, Jiangsu Cancer Hospital \& Jiangsu \\ Institute of Cancer Research \& Nanjing Medical University Affiliated Cancer Hospital, Nanjing, China). \\ Comment on: Ferro M, Vartolomei MD, Russo GI, et al. An increased body mass index is associated with a worse prognosis in patients administered \\ BCG immunotherapy for T1 bladder cancer. World J Urol 2018. [Epub ahead of print].
}

Submitted Oct 16, 2018. Accepted for publication Oct 20, 2018.

doi: $10.21037 /$ tau.2018.10.17

View this article at: http://dx.doi.org/10.21037/tau.2018.10.17

Bladder cancer is the tenth most commonly diagnosed cancer worldwide, with an estimated 549,000 new cases diagnosed in 2018 (1). Approximately $75 \%$ of patients present with non-muscle-invasive bladder cancer (NMIBC), which is confined to the mucosa (stage Ta, CIS) or submucosa (stage T1) (2). NMIBC is characterized by a high risk of disease recurrence and progression, with high-grade T1 tumours having the highest risks (3). Therefore, patients have to undergo frequent check-ups with cystoscopies and multiple intravesical treatments (2). Despite improvements in treatment such as single postoperative instillations of chemotherapy and contrastenhanced cystoscopy, recurrence rates are still high, indicating the need to identify additional prognostic factors that may support treatment, for example lifestyle factors.

Smoking is the most important risk factor for both NMIBC and muscle-invasive bladder cancer (MIBC). A recent meta-analysis showed that former and current smokers may be at a more than $20 \%$ higher recurrence risk compared to never smokers, but found no association with risk of progression (4). Overweight and obesity have been associated with risk and prognosis of other cancer types (5), but evidence for bladder cancer is still scarce and inconsistent. Three meta-analyses found that obesity was associated with a small increase in bladder cancer risk (6-8), with the most comprehensive doseresponse meta-analysis of 8 prospective cohort studies showing a $4.2 \%$ increased bladder cancer risk for each $5 \mathrm{~kg} / \mathrm{m}^{2}$ increase in body mass index (BMI) (pooled RR
$=1.04 ; 95 \%$ CI, $\left.1.01-1.07 ; \mathrm{I}^{2}=32.1 \% ; \mathrm{P}_{\text {heterogeneity }}=0.17\right)(7)$. However, the WCRF/AICR report on bladder cancer, based on 7 overlapping and 9 additional prospective cohort studies, judged the evidence for an association of obesity with bladder cancer risk as limited and inconclusive (9). Although results were in the same direction, they were not statistically significant (pooled RR per $5 \mathrm{~kg} / \mathrm{m}^{2}=1.03$; $95 \%$ CI, 0.97-1.09; $\mathrm{I}^{2}=55.1 \% ; \mathrm{P}_{\text {heterogeneity }}<0.01$ ). We recently investigated the association of $\mathrm{BMI}$ with clinical outcomes of bladder cancer by conducting a systematic review and meta-analysis, including twelve historical cohort studies and one prospective cohort study (10). Ten studies were performed in NMIBC and MIBC patients combined $(n=6)$ or exclusively in MIBC patients $(n=4)$, and showed inconsistent results. Three studies were conducted exclusively in NMIBC patients. Kluth et al. showed in $892 \mathrm{~T} 1$ high grade patients that $\mathrm{BMI} \geq 30$ vs. $<30 \mathrm{~kg} / \mathrm{m}^{2}$ was associated with an increased risk of recurrence and progression (11). This was confirmed by $\mathrm{Xu}$ et al. in 469 NMIBC patients with Ta and T1 tumours (12). In 338 NMIBC patients with Ta, T1, and Tis tumours, Wyszynski et al. found no statistically significant association for being overweight or obese compared to normal weight with the risk of recurrence, except when analyses were restricted to current smokers (13). A meta-analysis of these three studies showed that both overweight (pooled $\mathrm{HR}=1.29 ; 95 \%$ CI, $1.05-1.58 ; \mathrm{I}^{2}=0 \% ; \mathrm{P}_{\text {heterogeneity }}=0.41$ ) and obese NMIBC patients (pooled HR $=1.82 ; 95 \%$ CI, $1.12-2.95 ; \mathrm{I}^{2}=79 \% ; \mathrm{P}_{\text {heterogeneity }}=0.008$ ) had a statistically 
significantly increased risk of recurrence when compared to normal weight patients, while no association for progression was found (10). The recently published study entitled "An increased body mass index is associated with a worse prognosis in patients administered BCG immunotherapy for T1 bladder cancer" by Ferro et al. published in the World Fournal of Urology (14) adds to the current evidence.

The authors of this historical cohort study investigated the impact of BMI on recurrence-free, progression-free, cancer-specific, and overall survival in patients with highrisk NMIBC. They included 1,046 primary T1 highgrade NMIBC patients from 13 academic institutions who underwent transurethral resection of the bladder tumour (TURBT) and Bacillus Calmette-Guérin (BCG) treatment with maintenance according to the European Association of Urology (EAU) recommendations. Pre-TURBT BMI was calculated, and patients were followed up every 3 months with cystoscopy and urinary cytology for recurrence and progression according to EAU guidelines. Recurrence was defined as the appearance of any tumour, and progression as MIBC during follow-up. In total, $65 \%$ of patients developed a recurrence (median follow-up 26 months, IQR $9-47$ months) and 30\% a progression (median followup 43 months, IQR 36-58 months). The authors found that overweight (BMI $25-29.9 \mathrm{~kg} / \mathrm{m}^{2}$ ) and obesity (BMI $\geq 30 \mathrm{~kg} / \mathrm{m}^{2}$ ) were associated with a 4.0 - and 5.3 -fold higher risk of recurrence, respectively, and a 2.5 -fold higher risk of progression, after adjustment for age, gender, tumour size, multifocality, and concomitant CIS. No statistically significant associations with cancer-specific and overall survival were observed.

Strength of this study was the inclusion of a large and homogeneous study population of primary T1 high-grade NMIBC patients treated with BCG. Most other studies on BMI and bladder cancer prognosis conducted to date included heterogeneous study populations with different stages and grades of bladder cancer, but did not perform subgroup analyses by tumour stage and grade (10). Since the association of BMI with risk of recurrence and progression may differ by tumour stage and grade, true associations may possibly have been masked.

Similar to the other three studies on BMI and NMIBC prognosis, this study suffered from some limitations. As acknowledged by the authors, the study may be subject to selection bias, as it is multicentric and retrospective with different treatment patterns across centers, no centralized pathological evaluation, and involvement of various different surgeons. The authors did not clearly describe how patients were selected. It was also unclear how information on pre-TURBT BMI was collected, and whether BMI was self-reported or measured. Information on type 2 diabetes, which is common in obese patients and may be independently associated with NMIBC outcomes $(12,15,16)$, was not available. Although the authors report that a larger proportion of overweight and obese patients were current smokers, and smoking status has also been associated with risk of recurrence (4), statistical analyses were not adjusted for smoking. Information on post-diagnosis BMI or intentional weight loss was lacking. Thus, the authors' advice that subjects with $\mathrm{BMI} \geq 25 \mathrm{~kg} / \mathrm{m}^{2}$ should be invited to undergo a weight loss program to reduce their risk of recurrence and progression seems preliminary. Overweight and obese NMIBC patients typically suffer from other comorbidities for which weight loss may be beneficial. However, prospective cohort studies with multiple postdiagnosis BMI measurements or randomized weight loss trials are needed to investigate whether weight loss is indeed associated with reduced risk of NMIBC recurrence and progression. Also, information on other measures of obesity or body composition needs to be collected, such as waist circumference or waist-to-hip ratio, since abdominal obesity may confer the highest risk.

Potential mechanisms underlying the association of overweight and obesity with increased risk of recurrence and progression risk in NMIBC patients are still unclear. These may include systemic and local changes induced by overweight and obesity, such as altered levels of insulin, insulin-like growth factor-1, leptin, adiponectin, steroid hormones, and cytokines (17-22). Interestingly, markers of systemic inflammatory response, in particular the neutrophil-to-lymphocyte ratio, have been associated with increased risk of recurrence and progression NMIBC patients in the current (23) and other studies (24). Also, potential difficulties in performing a high quality (complete) transurethral resection of the bladder may play a role, particularly for obese patients $(11,12)$.

In conclusion, although it seems advisable for NMIBC patients to attain or maintain a healthy body weight, it should be kept in mind that available evidence is limited to four historical cohort studies with information on BMI only at diagnosis. Evidence from prospective cohort studies with multiple post-diagnosis BMI measurements or from randomized weight loss trials is needed before overweight NMIBC patients can be provided with evidence-based weight management advice to reduce their risk of 
recurrence or progression.

\section{Acknowledgements}

None.

\section{Footnote}

Conflicts of interest: The author has no conflicts of interest to declare.

\section{References}

1. Bray F, Ferlay J, Soerjomataram I, et al. Global cancer statistics 2018: GLOBOCAN estimates of incidence and mortality worldwide for 36 cancers in 185 countries. CA Cancer J Clin 2018;68:394-424.

2. Babjuk M, Bohle A, Burger M, et al. EAU Guidelines on Non-Muscle-invasive Urothelial Carcinoma of the Bladder: Update 2016. Eur Urol 2017;71:447-61.

3. Cambier S, Sylvester RJ, Collette L, et al. EORTC Nomograms and Risk Groups for Predicting Recurrence, Progression, and Disease-specific and Overall Survival in Non-Muscle-invasive Stage Ta-T1 Urothelial Bladder Cancer Patients Treated with 1-3 Years of Maintenance Bacillus Calmette-Guerin. Eur Urol 2016;69:60-9.

4. Hou L, Hong X, Dai M, et al. Association of smoking status with prognosis in bladder cancer: A meta-analysis. Oncotarget 2017;8:1278-89.

5. Colditz GA, Peterson LL. Obesity and Cancer: Evidence, Impact, and Future Directions. Clin Chem 2018;64:154-62.

6. Qin Q, Xu X, Wang X, et al. Obesity and risk of bladder cancer: a meta-analysis of cohort studies. Asian Pac J Cancer Prev 2013;14:3117-21.

7. Sun JW, Zhao LG, Yang Y, et al. Obesity and risk of bladder cancer: a dose-response meta-analysis of 15 cohort studies. PLoS One 2015;10:e0119313.

8. Zhao L, Tian X, Duan X, et al. Association of body mass index with bladder cancer risk: a dose-response meta-analysis of prospective cohort studies. Oncotarget 2017;8:33990-4000.

9. World Cancer Research Fund/International American Institute for Cancer Research. Continuous Update Project Report: Diet, Nutrition, Physical Activity and Bladder Cancer. 2015. Available online: www.wcrf.org/bladdercancer-2015

10. Westhoff E, Witjes JA, Fleshner NE, et al. Body mass index, diet-related factors, and bladder cancer prognosis: a systematic review and meta-analysis. Bladder Cancer 2018;4:91-112.

11. Kluth LA, Xylinas E, Crivelli JJ, et al. Obesity is associated with worse outcomes in patients with T1 high grade urothelial carcinoma of the bladder. J Urol 2013;190:480-6.

12. Xu T, Zhu Z, Wang X, et al. Impact of body mass on recurrence and progression in Chinese patients with Ta, T1 urothelial bladder cancer. Int Urol Nephrol 2015;47:1135-41.

13. Wyszynski A, Tanyos SA, Rees JR, et al. Body mass and smoking are modifiable risk factors for recurrent bladder cancer. Cancer 2014;120:408-14.

14. Ferro M, Vartolomei MD, Russo GI, et al. An increased body mass index is associated with a worse prognosis in patients administered BCG immunotherapy for T1 bladder cancer. World J Urol 2018. [Epub ahead of print].

15. Hwang EC, Kim YJ, Hwang IS, et al. Impact of diabetes mellitus on recurrence and progression in patients with non-muscle invasive bladder carcinoma: a retrospective cohort study. Int J Urol 2011;18:769-76.

16. Rieken M, Xylinas E, Kluth L, et al. Association of diabetes mellitus and metformin use with oncological outcomes of patients with non-muscle-invasive bladder cancer. BJU Int 2013;112:1105-12.

17. Hopkins BD, Goncalves MD, Cantley LC. Obesity and Cancer Mechanisms: Cancer Metabolism. J Clin Oncol 2016;34:4277-83.

18. Iyengar NM, Gucalp A, Dannenberg AJ, et al. Obesity and Cancer Mechanisms: Tumor Microenvironment and Inflammation. J Clin Oncol 2016;34:4270-6.

19. Yuan SS, Chung YF, Chen HW, et al. Aberrant expression and possible involvement of the leptin receptor in bladder cancer. Urology 2004;63:408-13.

20. Metalli D, Lovat F, Tripodi F, et al. The insulin-like growth factor receptor I promotes motility and invasion of bladder cancer cells through Akt- and mitogen-activated protein kinase-dependent activation of paxillin. Am J Pathol 2010;176:2997-3006.

21. Ishii N, Wei M, Kakehashi A, et al. Enhanced Urinary Bladder, Liver and Colon Carcinogenesis in Zucker Diabetic Fatty Rats in a Multiorgan Carcinogenesis Bioassay: Evidence for Mechanisms Involving Activation of PI3K Signaling and Impairment of p53 on Urinary Bladder Carcinogenesis. J Toxicol Pathol 2011;24:25-36.

22. Hariharan N, Ashcraft KA, Svatek RS, et al. Adipose Tissue-Secreted Factors Alter Bladder Cancer Cell Migration. J Obes 2018;2018:9247864. 
23. Vartolomei MD, Ferro M, Cantiello F, et al. Validation of Neutrophil-to-lymphocyte Ratio in a Multi-institutional Cohort of Patients With T1G3 Non-muscle-invasive Bladder Cancer. Clin Genitourin Cancer 2018;16:445-52. 24. Vartolomei MD, Porav-Hodade D, Ferro M, et al.

Cite this article as: Vrieling A. The association of BMI with risk of recurrence and progression in patients with non-muscleinvasive bladder cancer. Transl Androl Urol 2018;7(Suppl 6):S702S705. doi: 10.21037/tau.2018.10.17
Prognostic role of pretreatment neutrophil-to-lymphocyte ratio (NLR) in patients with non-muscle-invasive bladder cancer (NMIBC): A systematic review and meta-analysis. Urol Oncol 2018;36:389-99. 